\title{
Force and Deformation Analysis of Rails in Electromagnetic Launch
}

\author{
Mengyang Cuil ${ }^{1}$, Xuezhi Wang ${ }^{1}$, Songyi $\mathrm{Liu}^{2}$, Juan Bai ${ }^{1}$, Lei Bao ${ }^{1}$ and Qingrong \\ Chen ${ }^{1}$ \\ ${ }^{1}$ Air and Missile Defense College, Air Force Engineering University, Xi'an Shanxi, China; \\ ${ }^{2}$ China Satellite Launch Measurement and Control System Department, Beijing, China
}

Keywords: Electromagnetic rail launcher, Volume force density, Multi physical field coupling, Stress distribution.

\begin{abstract}
In order to realize the safe and continuous launching, the launching process of the electromagnetic rail launcher was simulated and analyzed, and the force and deformation mechanism of the rail and armature were obtained. Volume force density, current and magnetic field distribution data of different parts of rails and armature obtained by Maxwell simulation, and the data were imported into STATC Structural module of ANASYS Workbench, and the electromagnetic-stress multi-physical field coupling model was established, which can more accurately obtain the electromagnetic force and extrusion stress of rail during launching, as well as the deformation caused by them. The analysis of the force and deformation of the rail during the movement of armature can provide some reference for the selection of rail materials and the setting of parameters.
\end{abstract}

\section{Introduction}

Electromagnetic rail launcher is a new concept kinetic energy weapon, which uses the strong magnetic field produced by the high current flowing through the rail and armature to accelerate the armature to the exit under the action of Lorentz force and eject it. Due to the fast speed of armature movement, in order to ensure that armature and rail alrails have good contact conditions, it is necessary to apply appropriate pre-tightening force to rail. During the launching process, the armature is subjected to electromagnetic force and deformed, which will squeeze the contact rail surface. Because of the strong current flowing in different directions, the rail will also have a greater repulsion force. Therefore, to ensure that the rail is in a relatively stable working state and has a long service life is an important research direction of electromagnetic rail launching technology ${ }^{[1-2]}$.

Many scholars have studied the problem of rail deformation during launch. In document [3], the rail of electromagnetic launcher is simplified as a simply supported beam on elastic foundation under moving load to analyze its force and deformation; in document [4], the rail and wall of square-caliber electromagnetic launcher are simplified as double-layer elastic foundation beam to analyze the dynamic response of the rail and wall plate; in document [5], the control equation of the rail simplified as elastic foundation beam is transformed by Fourier transformation. The dynamic response equation of the rail during launching is derived, and the effects of geometric parameters of the rail, the strength of the input current and the elastic characteristics of the outer layer on the dynamic response of the rail are analyzed.

In this paper, the simulation model of quadrupole electromagnetic rail launcher is established by Maxwell electromagnetic analysis software. The electromagnetic field distribution of the rail launcher is analyzed after the current is applied. The volume force density of each part of the rail is obtained and the data are imported into the Static Structural static structure analysis module of ANASYS Workbench. The deformation of armature and rail and the distribution of equivalent stress are obtained by simulation.

\section{RAIL Force Analysis Model}

The structure of the electromagnetic rail launcher is shown in Fig. 1. Under the action of the strong 
magnetic field produced between the rails, the armature is subjected to the strong Lorentz force to slide along the rails. The armature will squeeze the rail in the course of moving along the rail. At the same time, the rail will be repulsed by the adjacent rail, as well as the pre-tightening force generated by the outer layer on the rail. The force acting on the rail is shown in Fig. 2.

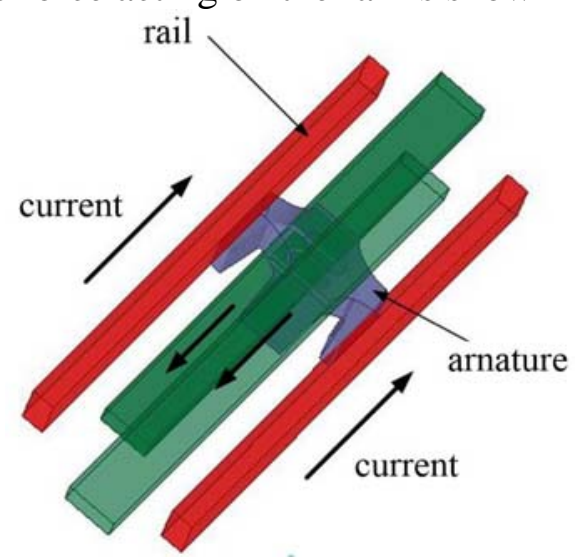

Figure 1. Structural model of electromagnetic launcher

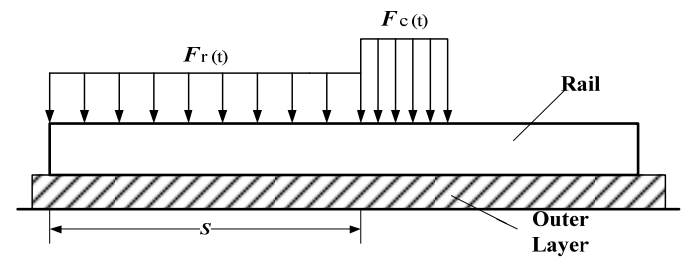

Figure 2. Force sketch of rail

In the stress analysis sketch of the rail, there are no constraints at both ends of the rail, and the outer layer provides elastic support for the rail. The dynamic governing equation of the rail ${ }^{[7]}$ is:

$$
E I \frac{\partial^{4} w(x, t)}{\partial x^{4}}+k w(x, t)+\rho A \frac{\partial^{2} w(x, t)}{\partial t^{2}}=q(x, t)
$$

In the formula above, $w(x, t)$ is the transverse deformation of rail, $E$ is the Young's modulus of rail material, I is the moment of inertia of rail cross-section, $E I$ is the flexural rigidity of rail, $k$ is the elastic coefficient of outer support, $\rho$ is the material density of rail, $A$ is the cross-section area of rail, $q(x, t)$ is the load of rail.

Fourier transforms of formulas (1) on $x, t$ are carried out, and the results are as follows:

$$
E I \alpha^{4} \bar{w}+k \bar{w}-\rho A \beta^{2} \bar{w}=\bar{q}(\alpha, \beta)
$$

Among them, $\alpha, \beta$ are integral vectors in Fourier transform, which can be concluded as follows:

$$
\bar{w}=\frac{\bar{q}(\alpha, \beta)}{E I \alpha^{4}+k-\rho A \beta^{2}}
$$

The inverse Fourier transform of equation (3) can be obtained as:

$$
w(x, t)=\frac{1}{4 \pi^{2}} \int_{-\infty}^{+\infty} \int_{-\infty}^{+\infty} \frac{\bar{q}(\alpha, \beta) e^{i(\alpha x+\beta t)}}{E I \alpha^{4}+k-\rho A \beta^{2}} \mathrm{~d} \alpha \mathrm{d} \beta
$$

That is, the transverse deformation of the rail during launching. 
Table 1. Calculation parameters

\begin{tabular}{|c|c|c|c|}
\hline Parameter/Unit & value & Parameter/Unit & value \\
\hline $\mathrm{a} / \mathrm{mm}$ & 50 & $\mathrm{~L} / \mathrm{mm}$ & 2000 \\
\hline $\mathrm{b} / \mathrm{mm}$ & 110 & $\mathrm{k} / \mathrm{MPa}$ & 4 \\
\hline $\mathrm{E} / \mathrm{GPa}$ & 14 & $\rho / \mathrm{kg} / \mathrm{m}^{3}$ & 8400 \\
\hline $\mathrm{d} / \mathrm{mm}$ & 300 & $I / \mathrm{mm}^{3}$ & $2.5 \times 10^{7}$ \\
\hline
\end{tabular}

\section{Analysis of Rail-related Forces}

The main stresses of the rail are: the compressive stress caused by the deformation of armature movement; the repulsive force between the rails; and the pre-tightening force of the outer layer of the electromagnetic rail launcher on the rail. The contact stress between armature and rail is mutual. By analyzing and calculating the force of electromagnetic force perpendicular to the contact surface of armature, the extrusion stress of armature on rail can be obtained.

\section{Interaction between Rails}

Due to the obvious skin effect of electric current on the orbit and armature during the launching process, the hypothesis is made as follows: 1 . Current has a certain skin depth on the inner surface of the rail; 2. Current concentrates on the central line of skin depth; 3. In practical application, the current section of the armature tail is shorter than that of the rail, so its exclusion effect on the rail is neglected.

Without considering the skin effect, we assume that the current is uniformly distributed in the rail. The cross-section length of the rail is $a$ and the width is $b$. Taking the vertical paper facing inward is the Z-axis positive direction, which is also the movement direction of the armature. The current direction in the rail is shown in Figure 3, and the four rails are numbered. The rails through which current flows is considered to consist of innumerable electrified wires, according to Biot-Safar's law,

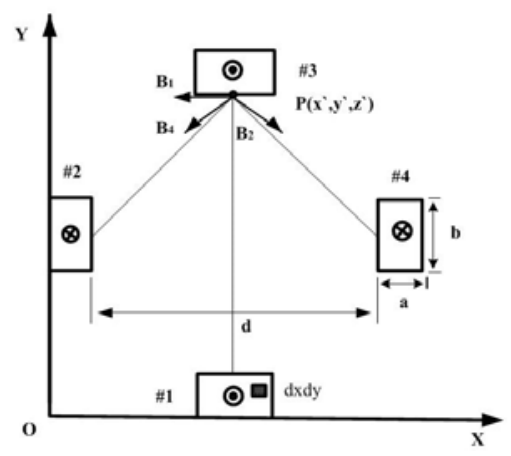

Figure 3. A schematic diagram of the magnetic field generated by the current in the rail

$$
d \boldsymbol{B}=\frac{\mu_{0}}{4 \pi} \frac{I d \boldsymbol{I} \times \boldsymbol{R}}{\left|\boldsymbol{R}^{3}\right|}
$$

the current element at any point on the rail 1 is $\frac{I}{a b} d \sigma \boldsymbol{i}=\frac{I}{a b} d x d y \boldsymbol{k}$, and the magnetic field intensity at the analysis point $P\left(x^{\prime}, y^{\prime}, z^{\prime}\right)$ on the rail 3 is

$$
d \boldsymbol{B}_{1}=\frac{\mu_{0}}{4 \pi} \frac{I d x d y \boldsymbol{k} \times \boldsymbol{R}}{a b\left|\boldsymbol{R}^{3}\right|}
$$

In the formula $\mu_{0}$ is the permeability in vacuum, $\boldsymbol{R}=\left(x-x^{\prime}\right) \boldsymbol{i}+\left(y-y^{\prime}\right) \boldsymbol{j}+\left(z-z^{\prime}\right) \boldsymbol{k},(x, y, z)$ is the coordinates of the center point of the current element, then there are

$$
|\boldsymbol{R}|=\sqrt{\left(x-x^{\prime}\right)^{2}+\left(y-y^{\prime}\right)^{2}+\left(z-z^{\prime}\right)^{2}}
$$


Regular Formula (2) is expressed in vector coordinates as

$$
d \boldsymbol{B}_{1}=\frac{\mu_{0} I}{4 \pi} \frac{d x d y}{a b\left|\boldsymbol{R}^{3}\right|}\left[\boldsymbol{k} \times\left(x-x^{\prime}\right) \boldsymbol{i}+\boldsymbol{k} \times\left(y-y^{\prime}\right) \boldsymbol{j}\right]
$$

Integrating formula (4) to obtain the magnetic induction intensity of current at $P$ point in rail 1

$$
\boldsymbol{B}_{1}=\int_{0}^{a} \int_{0}^{b} \frac{\mu_{0}}{4 \pi} \frac{I}{a b\left|\boldsymbol{R}^{3}\right|}\left[\boldsymbol{k} \times\left(x-x^{\prime}\right) \boldsymbol{i}+\boldsymbol{k} \times\left(y-y^{\prime}\right) \boldsymbol{j}\right] d x d y
$$

Similarly, it can be obtained that the magnetic field intensity produced by any rail at point $P$ is as follows:

$$
\boldsymbol{B}_{i}=\int_{0}^{a} \int_{0}^{b} \frac{\mu_{0}(-1)^{i+1}}{4 \pi} \frac{I}{a b\left|\boldsymbol{R}^{3}\right|}\left[\boldsymbol{k} \times\left(x-x^{\prime}\right) \boldsymbol{i}+\boldsymbol{k} \times\left(y-y^{\prime}\right) \boldsymbol{j}\right] d x d y
$$

According to the vector superposition principle of magnetic field, it can be concluded that the magnetic field intensity produced by the current in the rail at point $\mathrm{P}$ is

$$
\begin{array}{r}
\boldsymbol{B}=\sum_{i=1}^{4} \boldsymbol{B}_{i}=\frac{\mu_{0} I}{4 \pi} \sum_{i=1}^{4} \frac{(-1)^{i+1}}{a b\left|\boldsymbol{R}^{3}\right|} \int_{0}^{a} \int_{0}^{b}\left[\boldsymbol{k} \times\left(x-x^{\prime}\right) \boldsymbol{i}+\boldsymbol{k} \times\left(y-y^{\prime}\right) \boldsymbol{j}\right] d x d y \\
(i=1.2 .4)
\end{array}
$$

According to the deduction formula of electromagnetic force $\mathrm{d} F=I d l \times B$, it can be concluded that the electromagnetic repulsion force of the other three rails to rail 3 is

$$
F_{1}=\int_{0}^{l(t)} \int_{0}^{a} \int_{0}^{b} \frac{I}{a b} \times B d x d y d l k
$$

Similarly, the repulsion force between the other three rails can be obtained.

\section{Contact force between Armature and Rail}

When the current flows through the armature, the Joule effect will be more obvious because the current amplitude is larger, and more heat will be generated on the armature. For convenience of calculation, we assume that the current on armature is approximately uniform. According to Joule-Lenz theorem, under the action of strong current, the heat generated by armature is as follows

$$
Q=0.86 I^{2} R^{\cdot}
$$

Among them, 0.86 is the thermal equivalent of $1 \mathrm{w} / \mathrm{h}$ and $R^{\bullet}$ is the resistance of armature.

Because the launching process takes a relatively short time, we neglect the time-varying temperature on the armature. The temperature distribution on the armature is

$$
T=T_{f}+\frac{Q b^{2}}{8 \lambda_{T}}\left[1+\frac{4 \lambda_{T}}{b \boldsymbol{\alpha}_{F}}-4\left(\frac{y}{d}\right)^{2}\right]
$$

Among them, $T_{f}$ is the temperature of adjacent medium of armature, $\lambda_{T}$ is the thermal conductivity of armature material, $\boldsymbol{\alpha}_{F}$ is the surface heat transfer coefficient of armature material, $d$ is the armature width.

The thermal strain of armature is

$$
\boldsymbol{\varepsilon}_{T}=\frac{1}{d} \int_{-d / 2}^{d / 2} \boldsymbol{\alpha}_{T} T d z
$$

The resultant force of armature acting on rail is

$$
F_{2}=l_{d} h^{\bullet} E_{T} \boldsymbol{\varepsilon}_{T}=\frac{Q b h^{\bullet} E_{T} l_{d} \boldsymbol{\alpha}_{T}}{6}\left[\frac{b}{\lambda_{T}}+\frac{6}{\boldsymbol{\alpha}_{F}}\right]
$$

Among them, $l_{d}$ is the size of $\mathrm{Z}$ axis of armature, $E_{T}$ is the elastic modulus of rail material, $h^{\bullet}$ is 
the height of armature, $\boldsymbol{\alpha}_{T}$ is the linear expansion coefficient.

\section{Simulation and Analysis}

In the Ansys Maxwell electromagnetic analysis software, the simulation model of the electromagnetic rail launcher is established. The material of the rail is copper alloy, and the armature material is aluminum alloy. Other parameters of the launcher are shown in Table 2.

Table 2. The parameters of the rail launcher

\begin{tabular}{|c|c|}
\hline Parameter & value \\
\hline Caliber & $300 \mathrm{~mm} \times 300 \mathrm{~mm}$ \\
\hline Rail length, width and height & $2000 \mathrm{~mm} \times 110 \mathrm{~mm} \times 50 \mathrm{~mm}$ \\
\hline Amplitude of current & $300 \mathrm{kA}$ \\
\hline Armature thickness & $50 \mathrm{~mm}$ \\
\hline $\begin{array}{c}\text { Initial positive pressure on contact } \\
\text { surface }\end{array}$ & $60 \mathrm{~N}$ \\
\hline
\end{tabular}

\section{Modeling and Simulation}

The electromagnetic simulation in Maxwell can obtain the current distribution on the rail and armature, the magnetic field distribution in the bore and the volume force density of each part.

The vector diagram of the volume force density is shown in Fig. 5. From Fig. 5, we can see the force on different parts of the rail: the rail is repulsed outward and attracted inward; the position of the rail near the armature is subject to a larger electromagnetic force, and the direction is outward, which will make the rail deform.

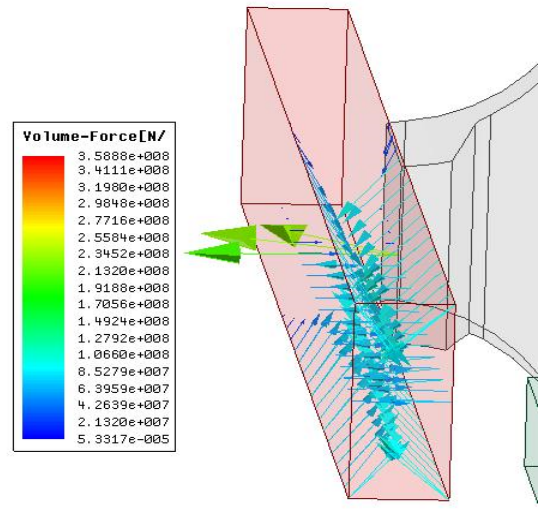

Figure 5. Volume force density distribution of rail

The electromagnetic rail launcher model and analysis results established in Maxwell are imported into Ansys Workbench, and the electromagnetic field-stress coupling simulation analysis model is established. The finite element analysis of stress and deformation is carried out in Static Structural static structural mechanics module. The deformation of rail and armature is simulated under the condition that fixed constraints are imposed on both ends of rail. When the armature moves to the middle position of the rail, the equivalent force and deformation of the armature and the rail are shown in figs. 6 and 7. With the displacement of armature, the magnetic field in bore will change, and the electromagnetic repulsion force between rails will gradually increase; the stress on the outer side of the rail is larger, while the stress in the middle part is smaller. 


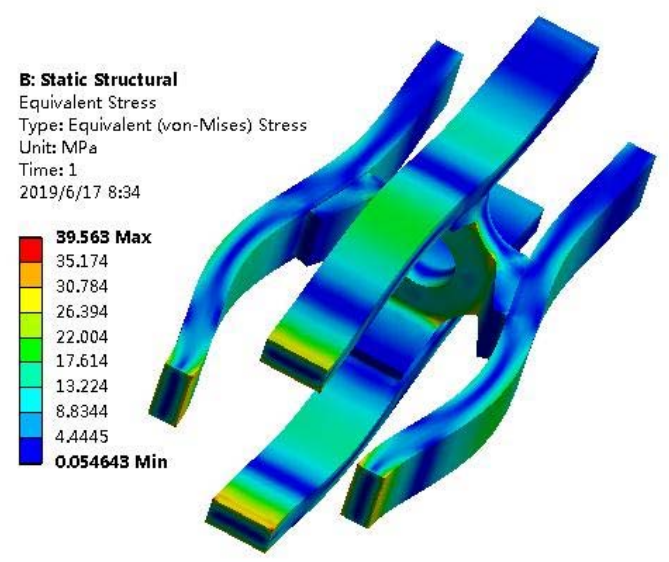

Figure 6. Equivalent stress distribution

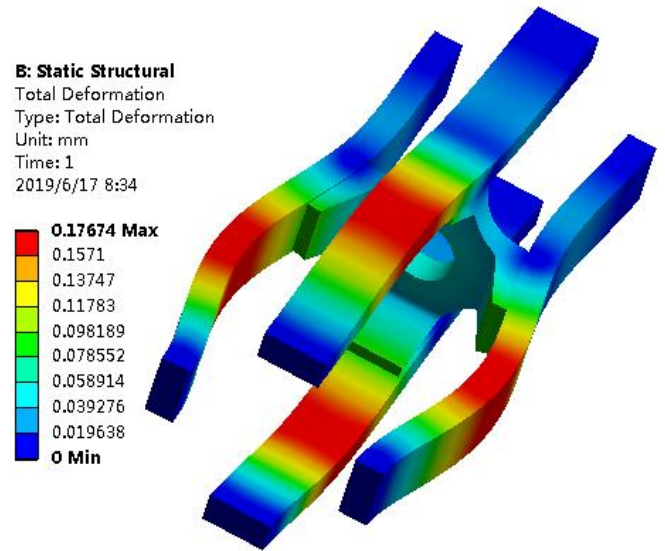

Figure 7. Deformation diagram of launcher

\section{Stress Analysis of Armature-Rail Interface}

In electromagnetic-stress coupling simulation, fixed constraints are imposed on the outer side of the four rails, and the rail and armature are meshed by sweeping method. When the displacement distance of armature is $1100 \mathrm{~mm}$, the stress distribution between armature and armature is shown in Fig. 8. The stress distribution on the rail is shown in figure 9. The extrusion stress of the armature on the rail increases gradually from the end to the shoulder, and the greater stress concentrates on the contact part of the armature shoulder. The stress value of the rail where the armature moved is small, which is the repulsive force between the rails. There is almost no stress distribution in the rail section without current.

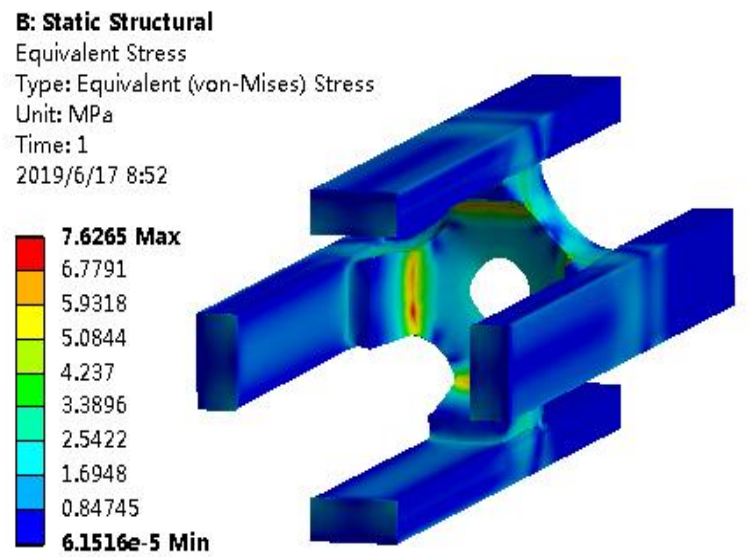

Figure 8. Equivalent stress distribution 


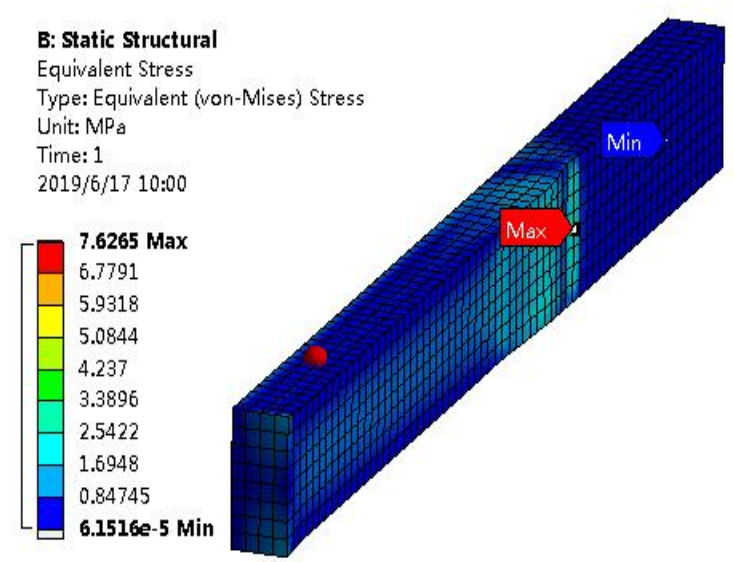

Figure 9. Equivalent force diagram of rail

\section{Conclusion}

In this paper, we establish the electromagnetic-stress multi-physical field coupling model of the electromagnetic rail launcher, and import the current, magnetic field and physical force density distribution of the launcher from Maxwell simulation into the Static Structural module of Workbench to obtain the stress and deformation of the rail and armature.

(1) Because of the extrusion of the armature and the repulsive force between the rails, the deformation deflection of the slipping area of the armature is larger than that of the non-slipping area.

(2) During the launching process, the tail wing of the armature will be opened outwards by strong electromagnetic force, and the material of the armature and rail can ensure good contact between the armature and the rail under the condition of wear resistance.

(3) The extrusion of armature to rail and the repulsive force between rails will lead to rail deformation. In order to ensure the launching accuracy, it is necessary to control the caliber change of the launcher. The shape, size and material of the rail cross section are all important factors affecting the flexural rigidity of the rail. Reducing the deflection amplitude of the rail can improve the stability of the rail launcher and help to realize the safe and continuous launching of the electromagnetic rail launcher.

\section{Acknowledgement}

This research was financially supported by the National Nature Science Foundation of China under Grant (61601503).

\section{References}

[1] Johnson A J,Haran T,Moon. Stress wave measurements in an electromagnetic launcher. 2008 14th Symposium on Electromagnetic Launch Technology . 2008:1-6

[2] Daneshjoo, Kamran,Rahimzadeh. Dynamic response and armature critical velocity studies in an electromagnetic railgun. IEEE Transactions on Magnetics . 2007,43(1):126-131

[3] Tzeng, Jerome T.,Sun, Wei. Dynamic response of cantilevered rail guns attributed to projectile/gun interaction-Theory. IEEE Transactions on Magnetics . 2007,43(1):207-213

[4] Zhiyong Yang,Gang Feng,Xinpeng Xue,Tao Shu.An Electromagnetic Rail Launcher by Quadrupole Magnetic Field for Heavy Intelligent Projectile[J]. IEEE Transactions on Plasma Science, 2017, 45(1): 1095-1099 
[5] Harry D.Fair ,Electromagnetic launch scienceand technology in the united states enters a new era. IEEE Transactions on Magnetics . 2005,41(1):158-164

[6] Li Jun ,Yan Ping, Yuan Wei-qun. Electromagnetic Railgun Technology and Its Development [J]. High Voltage Engineering, 2014, 40(4): 1052-1064

[7] Liu Zheng-guo, Tian Fu-qing. Structure dynamic characteristic analysis of electromagnetic rail launcher[J]. Journal of Ship Mechanics, 2010,14(10): 1158-1164

[8] Bai Xiang-zhong, He Wei. Deformation and stress analysis of rails and panels of railguns under armature's force [J]. Journal of Yanshan University ,2013,37(03):270-277.

[9] Tian Zhen-guo , Meng Xiao-yong . Dynamic Response of Composite Rail during Launch Process of Electromagnetic Railgun [J]. Acta Armament , 2017,38(04):651-657.

[10]Zhang Yong-sheng , Lu Jun-yong. Analysis of Force Load on the Rail in Rapid-fire Mode of Electromagnetic Rail Launcher [J]. Acta Armament,2018,39(03):618-624.

[11]Xie Guo-ping, Wang Guo-bo. Analysis of dynamic characteristic of rail system by moving load. [J]. Journal of Zhengzhou University, 2003, 24(1):24-27.

[12]Liu Ming, Shu Tao, Miao Haiyu, et al. Distribution characteristic of armature current in different orbit structures [J]. High Power Laser and Particle Beams, 2018, 30(5)

[13]Xue Xin-peng, Shu Tao. Design of multipole electromagnetic emitter for surface-to-air Missile[J].Journal of Projectiles, Rockets, Missiles and Guidance. 2017,37(02):27-31.

[14]Lei Bin, Li He. Rail deformation during Launching of an Electromagnetic Railgun [J]. Journal of Vibration and Stock . 2014,33(03):182-185.

[15]Chen Tie-ning. Dynamic response of electromagnetic railgun system due to armature movement[D]. Yanshan University ,2010. 